Chirurg 2013 $\cdot 84: 152$

DOI 10.1007/s00104-012-2461-0

Online publiziert: 27. Januar 2013

(c) Springer-Verlag Berlin Heidelberg 2013

J. Reibetanz • C.-T. Germer

Klinik für Allgemein-, Viszeral-, Gefäß- und Kinderchirurgie, Universitätsklinikum Würzburg

\title{
Kolon-J-Pouch vs. Koloplastiepouch nach tiefer Rektumchirurgie
}

\section{Funktionelle Langzeitergebnisse}

teten die postoperative Morbidität und Letalität.

\section{Ergebnisse}

(2012) Long-term functional results from a randomized clinical study of transverse coloplasty compared with colon J-pouch after low anterior resection for rectal cancer. Surgery. (Epub ahead of print). DOI 10.1016/j. surg.2012.08.012

\section{Hintergrund und Fragestellung}

Die Reservoirbildung nach tiefer anteriorer Rektumresektion zielt durch Verringerung von (Drang-)Inkontinenz und einer Reduktion der Stuhlfrequenz auf die Verbesserung der postoperativen Lebensqualität. Neben dem Kolon-J-Pouch (KJP) stellt der transverse Koloplastiepouch (TKP) eine alternative Pouchrekonstruktion insbesondere bei engem Becken dar, wobei man sich von letzterem eine ungestörtere Evakuation im Langzeitverlauf erhofft.

\section{Methode}

Zwischen 2000 und 2005 wurden in zwei spanischen Zentren Patienten mit Karzinomen der unteren beiden Rektumdrittel nach tiefer anteriorer Rektumresektion intraoperativ randomisiert und den Behandlungsarmen „KJP-“ oder „TKP-Anlage“ zugeteilt. Primärer Endpunkt der Studie war die Pouchfunktion zum Zeitpunkt 6 Monate und 3 Jahre nach Stomarückverlagerung, ausgedrückt durch Symptome der inkompletten Evakuation sowie Inkontinenzbeschwerden. Hierfür kamen bekannte Inkontinenzscores und Fragebögen zum Stuhlverhalten zur Anwendung. Sekundäre Endpunkte beinhal-
Von den inital 106 randomisierten Patienten konnten am Ende des Nachbeobachtungszeitraums die Daten von 86 Patienten (45 der KJP-Gruppe und 41 der TKPGruppe) ausgewertet werden. Bei einer Gesamtkomplikationsrate von 19,8\% war insbesondere die Rate an Anastomoseninsuffizienzen für beide Operationsverfahren identisch (jeweils je 1,9\%). Eine bei der Mehrzahl der Patienten initial postoperativ bestehende Inkontinenz besserte sich unabhängig von der Rekonstruktion im Verlauf deutlich. Ebenso reduzierte sich für beide Patientengruppen die Stuhlfrequenz pro Tag, wobei im Langzeitverlauf die Patienten der TKP-Gruppe in geringerem Maße unter nächtlichen Stuhlgängen litten. Die übrigen Dimensionen der krankheitsspezifischen Fragebögen (z. B. Laxanziengebrauch, Gefühl der unvollständigen Pouchentleerung etc.) diffemen, weder im 6-Monats- noch im 3-Jahres-Follow-up. Das krankheitsspezifische Überleben war für beide Patientengruppen vergleichbar.

\section{Diskussion und Fazit}

Die vorliegende prospektiv-randomisierte Studie evaluiert die funktionellen Aspekte des Pouches nach tiefer Rektumchirurgie im Langzeitverlauf. Am Ende des Nachbeobachtungszeitraums berichteten jeweils $95 \%$ der Patienten über ein zufriedenstellendes Stuhlverhalrierten nicht zwischen beiden Pouchfor- ten, was die Wertigkeit einer solchen Rekonstruktion unterstreicht. Signifikante Unterschiede zwischen KJP und TKP ließen sich nicht bestätigen, insbesondere führte - anders als erhofft - der TKP nicht zu einer niedrigeren Rate inkompletter Pouchevakuationen (TKP: 29\% vs. KJP: 33\%). Eine weitere kürzlich publizierte multizentrische Studie schlussfolgert beim Vergleich des KJP mit der Seitzu-End-Anastomose Ähnliches, wiederum ohne signifikante Bevorzugung eines Verfahrens in Bezug auf das funktionelle Ergebnis. Es scheint daher letztlich v. a. um die Vermeidung der geraden Anastomose zu gehen, weniger um die Art der Reservoirbildung im Speziellen. Die Evidenz für den KJP ist überzeugend; der TKP und die Seit-zu-End-Anastomose sind in funktioneller Hinsicht nicht minder günstig und evtl. als Rückzugsmethode bei widriger Lokalsituation (z. B. enges Becken) anzusehen.

\section{Korrespondenzadresse}

Univ.-Prof. Dr. C.-T. Germer

Klinik für Allgemein-, Viszeral-, Gefäß- und Kinderchirurgie, Universitätsklinikum Würzburg, Oberdürrbacher Str. 6, 97080 Würzburg Germer_c@klinik.uni-wuerzburg.de

Interessenkonflikt. Der korrespondierende Autor gibt für sich und seinen Koautor an, dass kein Interessenkonflikt besteht.

\section{Literatur}

1. Doeksen A, Bakx R, Vincent A et al (2012) J-pouch vs side-to-end coloanal anastomosis after preoperative radiotherapy and total mesorectal excision for rectal cancer: a multicentre randomized trial. Colorectal Dis 14:705-713 